\title{
INSECTOS FITÓFAGOS ASOCIADOS A Ficus benjamina L. Y A F. microcarpa L. (URTICALES: MORACEAE) EN LIMA, PERÚ
}

\section{PHYTOPHAGOUS INSECTS ASSOCIATED TO Ficus benjamina L. AND Ficus microcarpa L. (URTICALES: MORACEAE) IN LIMA, PERÚ}

\author{
Mónica Narrea-Cango ${ }^{1}$, Clorinda Vergara-Cobián² y Jenny Malpartida-Zevallos ${ }^{3}$
}

\begin{abstract}
Resumen
Los árboles de Ficus son ampliamente usados en el Perú con fines ornamentales en zonas urbanas y como cercos vivos en huertos y campos frutícolas; sin embargo, a pesar de haberse observado la presencia de insectos, son pocos los reportes de las especies que albergan estos árboles. Por esta razón, se realizó un reconocimiento para identificar los principales insectos fitófagos asociados a $F$. benjamina y a F. microcarpa, registrándose en este primer estudio, seis especies fitófagas y reportándose por primera vez para el Perú al diaspídido Chrysomphalus pinnulifer. Se detallan también los caracteres morfológicos diferenciales de estos insectos y los daños que ocasionan al Ficus, encontrándose que cuatro de las seis especies identificadas son también reportadas en frutales de importancia. Además, se confirma la alta especificidad de Gynaikothrips uzeli y $G$. ficorum hacia $F$. benjamina y $F$. microcarpa, respectivamente. Se concluye que los ficus estudiados son hospederos alternativos de plagas de importancia económica de frutales.
\end{abstract}

Palabras clave: Chrysomphalus pinnulifer, Gynaikothrips uzeli, Gynaikothrips ficorum, plagas de árboles frutales.

\begin{abstract}
Ficus trees are widely used in Perú as ornamental trees in urban areas as well as live fences in fields and fruit orchards. Nevertheless, despite the presence of insects, there are few reports of the species these trees support. For this reason a survey was conducted in order to identify the main phytophagous insects associated to $F$. benjamina and F. microcarpa. In this first paper, six phytophagous species are recorded and the diaspidid Chrysomphalus pinnulifer (Maskell) is reported for the first time in Perú. Differing morphological characters in these insects are detailed as well as damages they cause to Ficus. Five of the six insect pecies are also reported in fruitbearing trees. We also confirm the high specificity of Gynaikothrips uzeli and G. ficorum to F. benjamina and to F. microcarpa, respectively. We conclude that Ficus trees are alternative hosts to plagues in economic importance fruit-bearing trees.
\end{abstract}

Key words: Chrysomphalus pinnulifer, Gynaikothrips uzeli, Gynaikothrips ficorum, fruit trees pests.

\section{Introducción.}

El género Ficus (Moraceae) constituye uno de los principales géneros de angiospermas, con cerca de 800 especies de árboles, arbustos, hemi-epífitas, trepadoras $\mathrm{y}$ enredaderas que se encuentran en los bosques de todo el mundo, en especial en zonas tropicales y subtropicales (Frodin, 2004; Hamed, 2011).

En el Perú, De Orellana (2011), señala que desde fines del siglo XIX y principios del XX ya existían árboles de $F$. benjamina y $F$. microcarpa, ornamentando plazuelas y casas de Lima. No es, sino hasta el estudio de Wille (1943) sobre los insectos asociados a estos árboles, que logra registrar sólo una plaga: el "gusano peludo del ficus", Elysius sp.; (Lepidoptera: Arctiidae), aunque no señala explícitamente la especie hospedante. Posteriormente
Beingolea y Vásquez (1993) realizan un estudio bioecológico de la misma plaga en $F$. benjamina, actualizando el nombre científico del árctido como Ammalo helops Cramer.

$\mathrm{Al}$ igual que en el Perú, a nivel mundial, son pocos los estudios realizados sobre la fauna entomológica que albergan estas dos especies de Ficus. En los trabajos científicos existentes se mencionan a algunos insectos de la familia Aleyrodidae (Manzano et al., 1995), Pseudococcidae (Ben-Dov, 1994) y del orden Thysanoptera (Retana-Salazar, 2006). Entre los Thysanoptera se conoce el elevado grado de especificidad de Gynaikothrips ficorum (Marchal) en F. microcarpa y de Gynaikothrips uzeli (Zimmerman) en F. benjamina (Wheeler et al., 2006). 
Teniendo en cuenta que en el Perú existe la tendencia por sembrar estos árboles como cercos vivos en huertos y campos frutícolas, es de sumo interés establecer si los insectos que albergan constituyen plagas agrícolas. Por lo anterior, el presente trabajo tiene como finalidad determinar qué insectos fitófagos se desarrollan sobre árboles de $F$. benjamina y $F$. microcarpa sembrados con fines ornamentales $y$ agrícolas.

\section{Materiales y métodos.}

Entre febrero del 2003 y julio del 2005 se registraron visualmente y se muestrearon los insectos fitófagos que viven en $F$. benjamina y $F$. microcarpa en tres localidades de la provincia de Lima en la que estos árboles se encontraban formando parte de campos cultivados o como árboles ornamentales en parques públicos. Los sitios seleccionados, la Universidad Nacional Agraria La Molina (UNALM; $12^{\circ} 05^{\prime} 06^{\prime}$ ' S y 76 $56^{\prime} 30^{\prime \prime}$ O), Miraflores (12 ${ }^{\circ} 10^{\prime}$ $48^{\prime \prime}$ S y $76^{\circ} 55^{\prime} 28^{\prime \prime}$ O) y Villa María del Triunfo $\left(12^{\circ}\right.$ $07^{\prime} 36^{\prime}$ ' S y $77^{\circ} 01^{\prime} 17^{\prime}$ ' O). La fase de muestreo en cada una de estas localidades consistió en la colecta mensual de 10 hojas de ramas infestadas provenientes de 10 árboles con síntomas de daño, de modo que se tenían en cada colecta 100 hojas. En cada una de estas localidades se seleccionaron, en forma mensual 10 árboles con síntomas de daño y con un esfuerzo de muestreo acumulado de 300 horas-colector. Las muestras de hojas fueron etiquetadas y remitidas al Laboratorio de Investigaciones del Museo de Entomología “Klaus Raven Büller” de la UNALM, y los ejemplares obtenidos fueron depositados en la colección entomológica de la misma institución. Para la identificación se realizaron micropreparados de los individuos adultos. Para el caso de áfidos se siguió la técnica propuesta por Martin (1983); para los diaspídidos se empleó la técnica mejorada de McKenzie (1956) y para los trips se siguió la técnica propuesta por Soto-Rodríguez \& Retana-Salazar (2003). En todos los montajes se empleó bálsamo de Canadá como medio de montaje, dejándose secar los micropreparados en posición horizontal en estufa a $50^{\circ} \mathrm{C}$ por dos semanas antes de su manipulación. La identificación se hizo con un microscopio modelo Olympus con micrómetro ocular $(0.001 \mathrm{~mm})$, usando las claves de identificación y de descripción de especies de Ben-Dov (1974, 1994), Blackman \& Eastop (2000), Voegtlin et al. (2003) y Wheeler et al. (2006), mismas que se usaron para confirmar las descripciones morfológicas. La descripción de los insectos adultos vivos, insectos en micropreparados y las características que permiten su identificación a nivel de campo y los daños ocasionados fueron detalladas. Además, para establecer la importancia económica de los insectos identificados y los hospederos que éstos atacan se consultó la literatura científica especializada. Finalmente, con el objetivo de conocer los hospederos alternativos a los insectos registrados en el apartado anterior, se hicieron colectas de insectos fitófagos y observaciones en los campos frutícolas de La Molina (UNALM), Huaral ( $11^{\circ} 29^{\prime}$

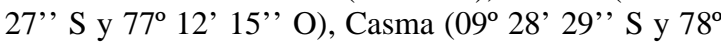
18'06”' O) y Piura (08 12 ' $10^{\prime \prime}$ ' S y $80^{\circ} 38^{\prime} 14^{\prime \prime}$ O), en enero, marzo y noviembre del 2005, para lo cual se escogieron 10 árboles con síntomas de infestación por insectos (estos es, con presencia de fumagina) de cada uno de los siguientes frutales: mandarina (Citrus unshiu Marc.; Rutaceae), naranja (C. sinensis L.), higuera (Ficus carica L.), mango (Mangifera indica L.; Anacardiaceae) y palto (Persea americana Mill.; Lauraceae), colectándose 10 hojas en cada uno de ellos a una altura de entre 0.8 a $1.60 \mathrm{~m}$, y entre las 10:00 y 11:00. El montaje e identificación de especímenes, se hizo, siguiendo la metodología descrita anteriormente.

Para el reporte final, sólo se tomó en cuenta los insectos fitófagos identificados hasta el nivel específico, no considerando insectos predatores o fitófagos que fueron identificados sólo hasta nivel genérico.

\section{Resultados.}

Se identificaron seis especies fitófagas, pertenecientes a los órdenes

Tabla 1. Lista de insectos fitófagos identificados en F. benjamina (F.b.) y F. microcarpa (F.m.) de tres localidades en Lima-Perú.

\begin{tabular}{|c|c|c|c|}
\hline Familia & Especie & Hospedero & Localidades $^{1}$ \\
\hline \multicolumn{4}{|c|}{ Orden Hemiptera } \\
\hline \multirow[t]{2}{*}{ Aphididae } & Toxoptera aurantii & F..b. & LM, VMT, MF \\
\hline & Greenidea ficicola & F.b., F.m..$^{2}$ & LM, VMT, MF \\
\hline \multirow[t]{2}{*}{ Diaspididae } & Ischnaspis longirostris & F.b. & LM, VMT, MF \\
\hline & Chrysomphalus pinnulifer ${ }^{3}$ & F.b. , F.m. & LM, VMT, MF \\
\hline \multicolumn{4}{|c|}{ Orden Thysanoptera } \\
\hline Phlaeothripidae & $\begin{array}{l}\text { Gynaikothrips ficorum } \\
\text { Gynaikothrips uzeli }\end{array}$ & $\begin{array}{l}\text { F.m. } \\
\text { F.b. }\end{array}$ & $\begin{array}{l}\text { LM, } \\
\text { LM, VMT, MF }\end{array}$ \\
\hline \multicolumn{4}{|c|}{${ }^{1}$ :LM: La Molina (Universidad Nacional Agraria La Molina) } \\
\hline \multicolumn{4}{|c|}{ VMT: Villa María del Triunfo } \\
\hline \multicolumn{4}{|c|}{ MF: Miraflores } \\
\hline \multicolumn{4}{|c|}{ 2: Nuevo hospedero en Perú } \\
\hline \multicolumn{4}{|c|}{ 3: Nuevo registro para el Perú } \\
\hline
\end{tabular}
Hemiptera y Thysanoptera. Tres de ellas se encontraron exclusivamente en $F$. benjamina: los hemípteros Toxoptera aurantii (Aphididae) e Ischnaspis longirostris (Diaspididae) y el tisanóptero Gynaikothrips uzeli (Phlaeothripidae); el trip $G$. ficorum fue registrado exclusivamente en $F$. microcarpa; y dos especies fueron registrados en ambas especies de Ficus: los hemípteros Greenidea ficicola (Aphididae) y Chrysomphalus pinnulifer (Diaspididae) (Tabla 1). 
Con excepción de G. ficorum (sólo encontrada en La Molina), todas las especies fueron registradas en las tres localidades estudiadas (Tabla 1). Por otro lado cuatro de estos seis fitófagos fueron encontrados en los frutales muestreados (Tabla 2).

Tabla 2. Lista de insectos fitófagos identificados en campos frutícolas de La Molina (LM), Huaral (HU), Casma (CA) y Piura (PI)

\begin{tabular}{|c|c|c|c|}
\hline Especie & Frutal hospedero & Localidades & Órgano atacado \\
\hline T. aurantii & Citrus unshiu, C. sinensis & LM, HU & Brotes y hojas jóvenes \\
\hline G. ficicola & C. unshiu ${ }^{1}$, C. sinensis ${ }^{1}$, & LM & Brotes \\
\hline G. ficicola & F. carica $^{1}$ & LM, HU & Hojas y fruto \\
\hline I. longirostris & Mangifera indica & HU, CA, PI & Hojas y fruto \\
\hline C. pinnulifer & Persea americana $^{1}$ & $\mathrm{HU}$ & Hojas \\
\hline C. pinnulifer & ${\text { F } \text { carica }^{1}}^{1}$ & LM & Hojas \\
\hline
\end{tabular}

${ }^{1}$ : Nuevos hospederos en Perú

La descripción de la morfología, así como del daño ocasionado en el Ficus por las especies identificadas se detalla a continuación:

Toxoptera aurantii (Boyer de Fonscolombe) (Hemiptera: Aphididae) (Figura1)

Ninfas y adultos de esta especie fueron encontradas en gran número, especialmente en las hojas jóvenes. Su forma ovalada, así como sus sifúnculos y cauda negros y abdomen variando de marrón a negro, permitieron distinguirlos fácilmente en las hojas verdosas de F. benjamina (Figura 1a).
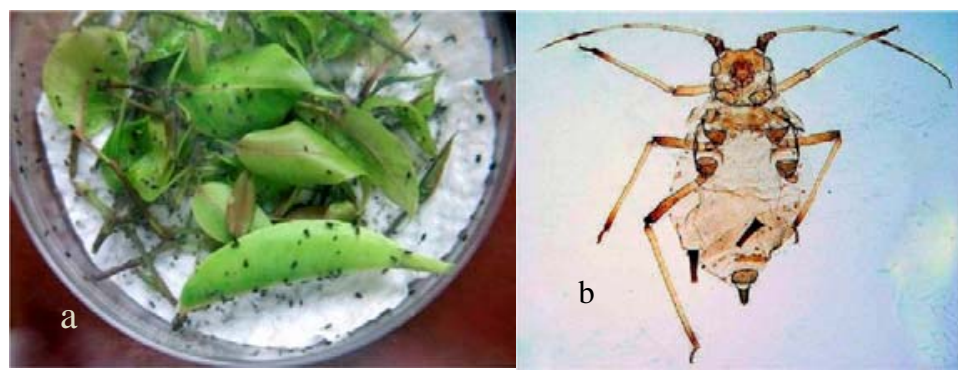

Figura 1. a) Colonias de Toxoptera aurantii en hojas de Ficus benjamina. b) Micropreparado de adulta áptera.

Caracterización morfológica (micropreparados). De tamaño pequeño, los especímenes ápteros (Figura 1b), midieron desde la cabeza hasta la cauda entre 1.525 y $2.289 \mathrm{~mm}$, de largo y entre 0.968 y $1.317 \mathrm{~mm}$ de ancho, siendo los sifúnculos más largos que la cauda, esta última con 16 a 18 setas En los alados se observó además la presencia de sólo una bifurcación de la vena media del ala anterior. Para la confirmación de la especie, se tomaron en cuenta la proporción del processus terminalis ( 3.5 a 5 veces) con relación a la base del VI segmento antenal, así como la presencia $\mathrm{y}$ número de rhinarias secundarias ( 2 a 8 en el III segmento antenal y ausentes en el IV y V).
Identificación a nivel de campo. T. aurantii puede confundirse con Aphis craccivora Koch y con $T$. citricida (Kirkaldi), pero se diferencian porque las dos últimas especies presentan dos bifurcaciones en la vena media del ala anterior.

Daños observados. Las colonias se encontraron en brotes y hojas jóvenes, principalmente en el envés. A niveles altos de infestación ocasionan el desarrollo de fumagina $y$ el debilitamiento $\mathrm{y}$ retraso en el crecimiento del árbol.

\section{Greenidea}

Takahashi (Hemiptera : Aphididae) (Figura 2)

Se encontraron en ramas y hojas de ambas especies de Ficus, los alados son marrón oscuros, algo más claros que los ápteros que son mayormente verdosos. Se evidencian fácilmente por su forma piriforme, sus numerosas y largas setas en el cuerpo y antenas, así como por sus sifúnculos largos, pilosos y distalmente divergentes (Figura 2a).

Caracterización morfológica (micropreparados). De tamaño mediano, los individuos ápteros midieron entre 2.010 y 2.254 mm de largo y entre 1.058 y 1.341 $\mathrm{mm}$ de ancho, con antenas largas de 1.841 a $2.047 \mathrm{~mm}$ y sifúnculos reticulados excepto en la parte apical; cauda con 8 setas. Las formas aladas (Figura 2b) midieron entre 1.880 y $2.115 \mathrm{~mm}$ de largo y entre 0.958 y $1.210 \mathrm{~mm}$ de ancho, con antenas largas de 1.608 a $1.923 \mathrm{~mm}$ y abdomen con bandas transversales dorsales; los sifúnculos cilíndricos débilmente reticulados y cauda al igual que en los ápteros con 8 setas y también reticulada.

Identificación a nivel de campo. Se diferencia de otros áfidos que atacan al ficus por la forma y color del cuerpo, setas y sifúnculos, así como por el desplazamiento rápido e inusual que tienen las ninfas y adultos. Podría confundirse con G. psidii, pero los ápteros de esta
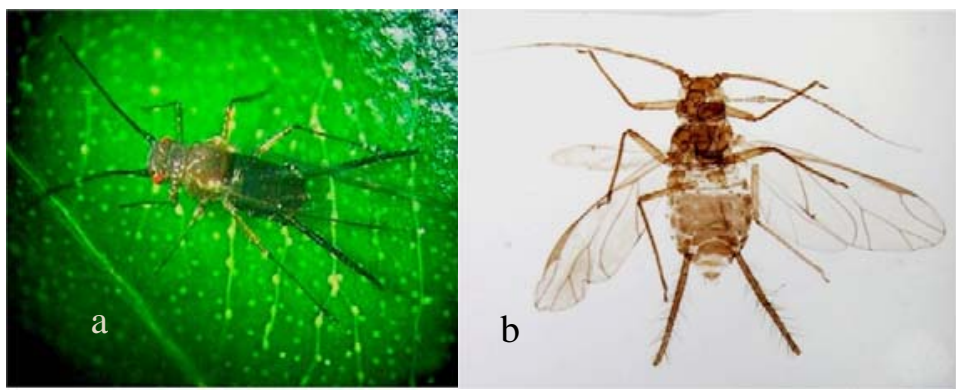

Figura 2. a) Adulto de Greenidea ficicola mostrando los sifúnculos largos y divergentes. b) Micropreparado de adulta alada, mostrando los sifúnculos pilosos. 
especie tienen los sifúnculos reticulados sólo en la base. Ambas especies son registradas en Ficus spp. y en Psidium guajava, pero G. psidi no está reportada en el Perú.

Daños observados. G. ficicola se encontró en tallos, ramas y hojas, siendo evidente su presencia por la fumagina que se desarrolla y el aspecto debilitado del árbol. En muchos de los árboles se encontraron en grandes colonias e incluso en hojas colindantes a hojas infestadas por T. aurantii.

Ischnaspis longirostris (Signoret) (Hemiptera: Diaspididae) (Figura 3)

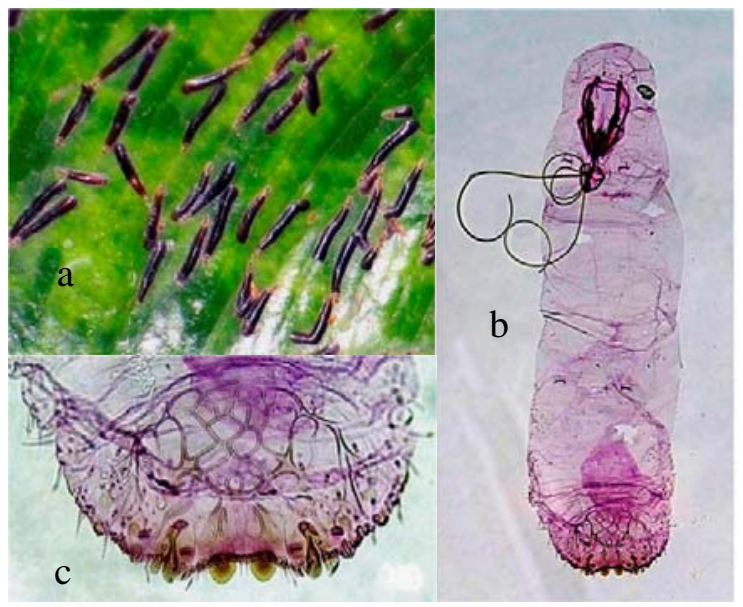

Figura 3. a) Colonia de Ischnaspis longirostris en hojas de $F$. benjamina. b) Micropreparado de adulto hembra. c) Detalle del pygidium reticulado.

Se encontraron principalmente en el haz de las hojas de $F$. benjamina, las escamas del adulto son rectas y convexas de color negro brillante a opaco, con la exuvia marginal y amarilla. Midieron entre $2.894 \mathrm{y}$ $3.125 \mathrm{~mm}$, de largo y entre 0.279 y $0.315 \mathrm{~mm}$ de ancho, es decir en promedio casi 11 veces más largas que anchas (Figura 3a). No se encontraron machos de esta especie.

Caracterización morfológica. En los micropreparados, el cuerpo de la hembra adulta (Figura 3b) midió entre $1.117 \mathrm{~mm}$ y $1.380 \mathrm{~mm}$, de largo y entre 0.270 y $0.292 \mathrm{~mm}$ de ancho, con una proporción promedio de 3.5 a 4.5 veces más largo que ancho. Tanto en el mesotórax, metatórax y en el I segmento abdominal se observaron ductos tuberculares. Para la confirmación de la especie se tomó en cuenta el pygidium (Figura 3c) el cual presentó el dorso esclerotizado en un patrón reticulado, con un par de lóbulos medios redondeados bien separados y un par de setas marginales entre ellos. El ano muy cerca al centro del pygidium.

Identificación a nivel de campo. La especie apropiadamente es llamada “escama hilo negro" por su forma alargada, delgada y de color oscuro, la cual es más fácil de observar cuando forma colonias numerosas. Puede confundirse con Lepidosaphes beckii (Newman) pero I. longirostris es más delgada y tiene una cresta media longitudinal muy evidente que no se encuentra en la "queresa coma”.

Daños observados. En ataques severos, se observó causando caída de hojas y debilitamiento general del árbol.

$$
\text { Chrysomphalus pinnulifer }
$$$$
\text { (Hemiptera: Diaspididae) (Figura 4) }
$$

(Maskell)
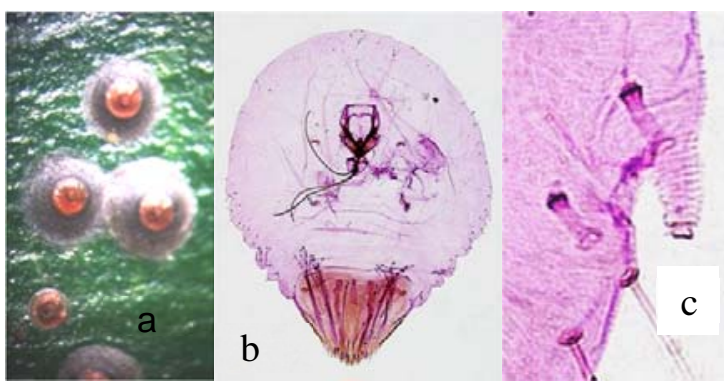

Figura 4.

a) Colonias de Chrysomphalus pinnulifer en Ficus microcarpa. b) Micropreparado de adulta hembra mostrando la forma piriforme y el pygidium. c) Detalle de los procesos laterales no espinosos.

Las escamas de hembras y machos (Figura 4a) se encontraron principalmente en el haz de las hojas ambas especies de Ficus con algunos pocos individuos en el envés. La escama de la hembra es circular, aplanada y delgada, con un diámetro entre $1.785 \mathrm{~mm}$ a $2.005 \mathrm{~mm}$, color marrón rojiza y con el margen blancuzco, con la exuvia central, amarillo a marrón, a veces blanco. La escama del macho es oval con una longitud entre 1.225 y 1.568 mm. El macho es más pequeño que la hembra, pero de color similar a ésta, pero se diferencia en que la exuvia es subterminal.

Caracterización morfológica. La hembra adulta (Figura 4b) tiene el mesotórax dilatado y piriforme, presenta todos los segmentos prepigidiales con menos de 5 conductos submarginales por lado. Como característica diferencial presenta a cada lado del III segmento un proceso no espinoso (Figura 4c). Tiene poros perivulvares $\mathrm{y}$ el pygidium es amplio, subtendido por un ángulo superior a $90^{\circ}$, con tres pares de lóbulos redondeados y el borde (entre los segmentos VI y VII) con numerosos macroconductos ordenados en doble o triple hilera.

Identificación a nivel de campo. En las hojas del ficus estas escamas se observaron fácilmente como pequeños discos aplanados color marrón.

Daños observados. Esta queresa se encontró atacando hojas jóvenes y viejas, produciendo caída de hojas y debilitamiento general del árbol.

Gynaikothrips ficorum (Thysanoptera: Phlaeothripidae) (Figura 5)

Adultos y ninfas se encontraron solo en $F$. microcarpa. No se observan a simple vista, sino que 
su presencia se detecta por las hojas dobladas a manera de estuche. Sólo se encontraron en brotes y hojas jóvenes, nunca en hojas maduras. Los adultos de ambos sexos son alados, marrones con tarsos y ápices de las tibias amarillos.

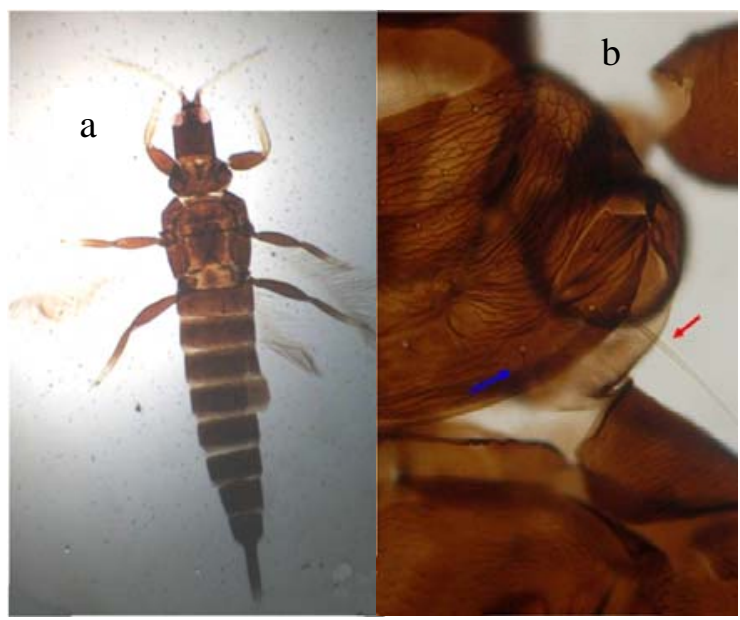

Figura 5. a) Micropreparado del adulto de Gynaikothrips ficorum. b) Detalle del pronotum mostrando la seta posteroangular (flecha azul) y la seta epimeral (flecha roja).

Caracterización morfológica. Los individuos (Figura. 5a) presentan las antenas con ocho segmentos, de los cuales los segmentos III al VII son amarillos y el VIII marrón claro, además el III tiene un sensorio y el IV tres; la longitud del cuerpo desde la cabeza hasta el final del abdomen, varió entre 2.965 y $3.380 \mathrm{~mm}$. Son estriados dorsalmente a excepción de las patas y el último segmento abdominal. La cabeza es alargada, las setas postoculares de ápice romo se extienden hasta el borde posterior del ojo. Pronoto esculturado con setas variables, la seta anteromarginal diminuta, las anteroangulares comúnmente bien desarrollados, las posteroangulares de una longitud promedio de $0.000927 \mathrm{~mm}$ mucho más cortas que las epimerales (longitud promedio $0.0146 \mathrm{~mm}$ ), observándose en todos los especímenes montados la proporción de 1:16 entre ellas (Fig. 5b). Suturas epimerales a menudo incompleta. El metanoto longitudinalmente reticulado.

Identificación a nivel de campo y daños observados. El daño característico que provoca en Ficus y que sirve además, para señalar su presencia es la formación de agallas y el doblez adaxial de la hoja que forma una especie de estuche, dentro del cual se desarrollan estos insectos.

\section{Gynaikothrips} uzeli

(Zimmerman)

\section{(Thysanoptera: Phlaeothripidae (Figura 6)}

Adultos y ninfas se encontraron solo en $F$. benjamina. La forma, color y características del cuerpo, son las mismas que describen a los adultos de
G. ficorum, excepto en el tamaño, pues las especies colectadas de $G$. uzeli, fueron más pequeñas, midiendo entre 2.589 y $2.913 \mathrm{~mm}$ de longitud.

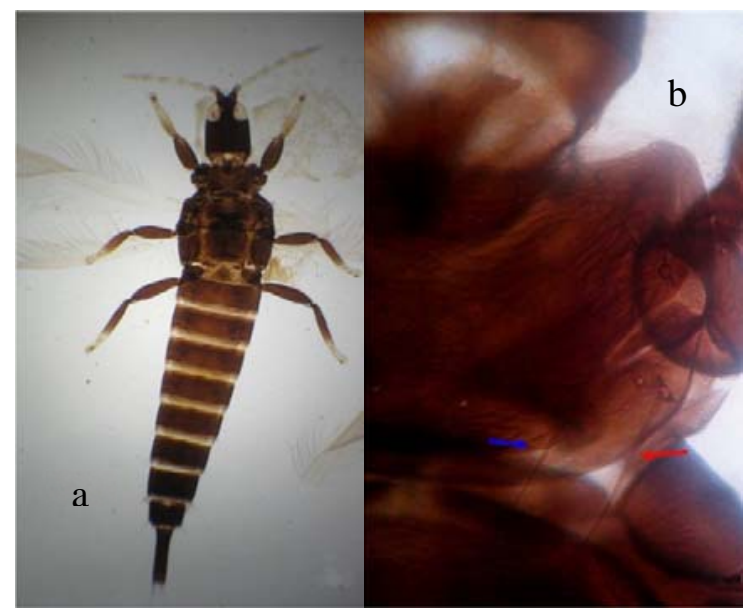

Figura 6. a) Micropreparado del adulto de Gynaikothrips uzeli. b) Detalle del pronotum mostrando la seta posteroangular (flecha azul) y la seta epimeral (flecha roja).

Caracterización morfológica. G. uzeli, (Fig. 6a) al igual que $G$. ficorum tiene el cuerpo estriado, con la cabeza alargada y con el pronoto esculturado, pero fue fácil su diferenciación por la longitud de las setas posteroangulares (largo promedio: $0.00854 \mathrm{~mm}$ ) casi tan largas a las epimerales (largo promedio: 0.0117 $\mathrm{mm})$; observándose en todos los especímenes montados la proporción de 1:1.37 entre estas setas (Fig. 6b).

Identificación a nivel de campo y daños observados. Igual a G. ficorum.

\section{Discusión.}

Todas las especies identificadas coincidieron con las descripciones de las claves y demás caracteres morfológicos diferenciales, aun cuando el tamaño no siempre correspondió a lo reportado por otros autores, tal vez porque la mayoría de los estudios taxonómico se realizan sobre hospederos agrícolas y no sobre ornamentales.

Así, la especie T. aurantii de nuestro estudió fue ligeramente más grande y con menor rango de setas en la cauda que lo señalado por Voegtlin et al. (2003), quienes indican que el rango de longitud es de 1.2 a $1.8 \mathrm{~mm}$ con 12 a 18 setas en la cauda. Esta especie la encontramos sólo en F. benjamina; sin embargo, es reportada en otras especies de Ficus, incluyendo al F. microcarpa y a la higuera (F. carica) (Holman, 2009). Con respecto a los frutales, nuestros reportes en mandarina (Citrus unshiu) y naranja (C. sinensis), confirman su afinidad por los cítricos que son considerados sus hospederos preferidos. Por su parte Cortez-Madrigal (2006) y Ripa et al. (2008) lo 
reportan también en cacao (Theobroma cacao) y palto (Persea americana).

Por otro lado, se observó que tanto los individuos ápteros como los alados de G. ficicola presentan un tamaño intermedio según lo reportado por Blackman y Eastop (2000), quienes precisan un rango de longitud 1.8 a $2.5 \mathrm{~mm}$ para las formas ápteras y de 2.15 a 2.28 mm para las aladas. Estos mismos autores señalan que los sifúnculos son reticulados excepto en la parte apical, pero no mencionan que esta reticulación se presenta también en la cauda, tal como se observó en este estudio. Sobre sus hospederos en América del Sur, Sousa-Silva et al. (2005) citan esta especie por primera vez en Brasil en F. microcarpa; mientras que Rubín de Celis et al. (2006), Sepúlveda et al. (2009) y La Rossa et al. (2011) reportan como su hospedero a F. benjamina en Perú, Colombia y Argentina, respectivamente. Sin embargo para Blackman y Eastop (2000), el rango de hospederos de esta especie es mucho más amplio, reportándola en más de 20 especies de Ficus, entre ellos $F$. benjamina y $F$. microcarpa, lo que es corroborado por nuestros resultados. En cuanto a los frutales, David et al. (2009) reportan la presencia de esta especie en Colombia en los guayabos (Psidium guajava), mientras que en este trabajo reportamos a la mandarina, la naranja y la higuera como nuevos hospederos en el Perú.

Las características del cuerpo y escama de $I$. longirostris no difiere en mucho con lo reportado por Alvarado (2011) quien en un estudio realizado en mango ( $M$. indica), encontró que las dimensiones promedio de la escama eran de $3.191 \mathrm{~mm}$ de largo por $0.281 \mathrm{~mm}$ de ancho, mientras que en el cuerpo registró $1.21 \mathrm{~mm}$ de largo por $0.269 \mathrm{~mm}$ de ancho. Esta especie es considerada polífaga, siendo reportada en más de 158 hospederos, entre ellos a $F$. benjamina, F. microcarpa y cultivos de importancia como el café (Coffea arabica L.; Rubiaceae), coco (Cocos nucifera L.; Areacaceae), chirimoya (Annona cherimolia Mill.; Annonaceae), los guayabos, el palto, la naranja y la lúcuma (Pouteria lucuma R\&L; Sapotaceae), entre otros (CABI, 2005), pero por nuestra parte sóo la encontramos en $F$. benjamina y en mango.

Los individuos colectados de $C$. pinnulifer, al igual que en el caso anterior, presentaron características de su cuerpo y su escama similar a lo reportados por otros autores (CABI, 2005). Esta especie es muy semejante a $C$. dictyospermi, pero es posible su diferenciación preliminar por el color, ya que $C$. pinnulifer es más oscura; sin embargo, fue necesario confirmar su identificación a través de micropreparados, teniendo en cuenta que según Culik et al. (2008), F . benjamina alberga a C. dictyospermi. En el presente trabajo también registramos como hospederos de $C$. pinnulifer a las dos especies de Ficus, al palto y a la higuera. A nivel mundial,
Williams \& Watson (1988) registran a Citrus sp. como uno de su hospederos.

Sobre los trips G. ficorum y G. uzeli son especies que son fácilmente detectadas en campo por la forma de las hojas donde se desarrollan que, según Meyer (1987), se debería a que estos trips, al raspar y succionar inyectan su saliva que tiene un efecto tóxico sobre las hojas. Ambos trips, a simple vista, sólo difirieron en el tamaño, siendo necesaria la realización de micropreparados para establecer su identidad. Según Retana-Salazar (2006), quien no establece diferencias en el tamaño del cuerpo, señala que la seta posteroangular de $G$. ficorum es menor o igual a 0.5 veces la longitud de la seta epimeral, mientras que en G. uzeli es casi tan desarrollada como la seta epimeral. En nuestros especímenes, la diferencia entre el tamaño de setas fue mucho más extrema, siendo por ello relativamente fácil establecer la identidad de cada uno de ellos y confirmar la relación altamente específica de $G$. ficorum y F. microcarpa, y de $G$. uzeli y $F$. benjamina, tal como lo señalan Wheeler et al. (2006). Esta especificidad es confirmada por Retana-Salazar (2006) al reportar que no pudo inducir el ataque de este trip en $F$. benjamina, a pesar de que repitió muchas veces el ensayo. Por su parte, Denmark et al. (2005) discrepan al mencionar que existen otras especies de Ficus como hospederos de G. ficorum, pero no precisan si completa su ciclo en estas especies. Estos últimos autores también señalan entre sus cultivos hospederos al tabaco (Nicotiana tabacum L.; Solanaceae), Citrus sp. y Eucalyptus sp. La literatura no reporta otros hospederos.

\section{Conclusiones.}

En este primer trabajo se reportan como insectos fitófagos de $F$. benjamina y $F$. microcarpa a $T$. aurantii, G. ficicola, I. longirostris, C. pinnulifer, G. ficorum y G. uzeli.

De las dos especies de áfidos, encontramos que $G$. ficicola fue reportada en el Perú sólo en $F$. benjamina. Esta especie tiene ahora como nuevos registros a $F$. microcarpa, la mandarina, la naranja y la higuera.

De los dos diaspídidos encontrados, I. longirostris es la más común, y en el presente estudio se registra también en el mango, mientras que $C$. pinnulifer, se reporta como nuevo registro para el Perú, siendo sus hospederos F . benjamina, F. microcarpa, el palto y la higuera.

Por su parte, la identificación de las dos especies de trips comprueba la alta especificidad de éstas con su hospedero.

En este estudio se demuestra que ambas especies de ficus son hospederos de plagas de importancia en frutales, condición que debe ser tomada en cuenta al momento de decidir su siembra como cercos vivos de fundos y al momento de implementar un programa de manejo de plagas en frutales. De las seis especies identificadas cuatro fueron encontradas en frutales de 
importancia y coincidentemente esas cuatro tienen como hospedero a $F$. benjamina, la especie de ficus más comúnmente sembrada.

La identificación previa de ambas especies de ficus fue un proceso importante en el estudio de las especies identificadas, pudiendo establecerse que $F$. benjamina acaso por ser el de más amplia distribución y el que alberga el mayor número de insectos fitófagos de importancia.

\section{Agradecimientos.}

Agradecemos al Blgo. Axel Retana, director del Programa Universitario de Desarrollo Agrario de la Universidad de Costa Rica, por sus importantes aportes al trabajo, y a Lourdes Huamán de la Facultad de Agronomía de la UNALM por su ayuda en la colección del material.

\section{Literatura citada.}

Alvarado J. 2011. Biología y Comportamiento de Ischnaspis longirostris, (Signoret) (Hemiptera: Diaspididae) en Mango (Mangifera indica L.) variedad Kent, bajo condiciones de Laboratorio. Tesis de maestría. Universidad Nacional Agraria La Molina.120pp.

Beingolea O. \& Vasquez G. 1993. Notas bioecologicas sobre el "gusano peludo del ficus" en Lima. Revista Per. Entomol., 36: 39-44

Ben-Dov Y. 1974. A revision of Ischnaspis Douglas with a description of a new allied genus (Homoptera: Diaspididae). Journal Entomology (B), 43:19-32.

1994. A Systematic Catalogue of the Mealybugs of the World (Insecta: Homoptera: Coccoidea: Pseudococcidae and Putoidae) with Data on Geographical Distribution, Host Plants, Biology and Economic Importance. Intercept Limited. Andover, UK.

Blackman R.L. \& Eastop V.F. 2000. Aphids on the World's Crops: An Identification and Information. Guide Chichester. Second edition. Department of Entomology. The Natural History Museum. London.

CABI. 2005. Crop Protection Compendium. CD-ROM databases. Wallingford, UK.

Cortez-Madrigal H. 2006. Efecto de coadyuvantes en Lecanicillium lecanii (Zimm.) Zare y Gams y su virulencia hacia Toxoptera aurantii Boyer. Revista Mexicana de Fitopatología. 24: 59-64.

Culik M., Martins D., Ventura J. \& Wolf V. 2008. Diaspididae (Hemiptera: Coccoidea) of Espírito Santo, Brazil. Journal of Insect Science. 8:17-22.

David R., Quiroz J., Yepes F. \& Smith A. 2009. Nuevo registro de Greenidea ficicola Takahashi (Hemiptera: Sternorrhyncha: Aphididae) en guayabo Psidium guajava (Myrtaceae) en Antioquia, Colombia. Revista Facultad Nacional de Agronomía, Medellín, 62(1): 4999-5002.

De Orellana J. 2011. La arquitectura del paisaje en la costa central del Perú y su sostenibilidad. Revista UniféConsensus -.16 (1): 183-213.

Denmark H., Thomas R. \& Funderburk E. 2005. Cuban Laurel Thrips, Gynaikothrips ficorum (Marchal) (Insecta: Thysanoptera: Phlaeothripidae). DPI Entomology Circular 59. Series of Featured Creatures from the Entomology and Nematology Department, Florida Cooperative Extension Service, Institute of Food and Agricultural Sciences, University of Florida. Florida. 4 pp.

Frodin, D.G. 2004. History and concepts of big plant genera. Taxon 53: 753-776.

Hamed M. A. 2011. Beneficial effect of Ficus religiosa Linn. on highfat induced hypercholesterolemia in rats. Food Chem.; 129: 162-170.

Holman, J. 2009. Host Plant Catalog of Aphids Palaearctic Region. Springer Science and Business Media B.V. New York. 1216 pp.

La Rossa, F., Vasicek, A. \& Paglioni, A. 2011. Primer registro de Greenidea ficicola Takahashi (Hemiptera: Aphididae) en Argentina. Revista de la Facultad de Ciencias Agrarias, 43(1): 247-252.

Manzano F., Carnero A., Pérez-Padrón F. \& Gonzáles A. 1995. Aleurodicus dispersus Russell (Homoptera, Aleyrodidae) una "mosca blanca" de importancia económica en Canarias, con especial referencia a la isla de Tenerife. Bol. San. Veg. Plagas. 21(1): 3-9.

Martin J. 1983. The identification of common aphid pests of tropical agriculture. Tropical Pest Management. 29: 395411.

McKenzie H. 1956. The armored scale insects of California. Bulletin of the California Insect Survey. 5: 81-83.

Meyer J. 1987. Plant Galls and Gall Inducers. Gebrüder Borntraeger. Berlin, Germany.

Retana-Salazar A. 2006. Variación morfológica del complejo Gynaikothrips uzeli-ficorum (Phlaeothripidae: Tubulifera). MES Costa Rica. 2006-1(1): 1-9.

Ripa R., Larral P. \& Rojas S. 2008. Manejo de Plagas en Paltos y Cítricos. Colección Libros INIA No 23. Instituto de Investigaciones Agropecuarias (INIA). Centro Regional de Investigación. Ministerio de Agricultura La Cruz, La Cruz, Chile.

Rubín de Celis V., Ortiz M. \& Barletta C. 2006. Greenidea ficicola Takahashi (Hemiptera: Aphididae) nuevo registro para Sudamérica. Revista Per. Entomol. 45: 105-107

Sepúlveda P., Ocampo L., Gaviria A. \& Rubio J. 2009. Trips (Thysanoptera) asociados a agallas a Ficus benjamina en la Región Central de Colombia. Revista Facultad Nacional de Agronomía-Medellín. 62: 50815087.

Soto-Rodríguez G. \& Retana-Salazar A. 2003. Clave ilustrada para los géneros de Thysanoptera y especies de Frankliniella presentes en cuatro zonas hortícolas en Alajuela, Costa Rica. Revista de Agronomía Costarricense (Costa Rica). 27(2): 55-68.

Sousa-Silva C., Brombal J. \& Ilharco A. 2005. Greenidea ficicola Takahashi (Hemiptera: Greenideidae), a new aphid in Brazil. Neotropical Entomology. 34(6): 10231024.

Voegtlin D., Villalobos W., Sánchez M., Saborio G. \& Rivera C. 2003. Guía de los áfidos alados de Costa Rica. Revista de Biología Tropical. 51(2): 22-38.

Wheeler C., Held D. \& Boyd D. 2006 Morphological differences between two gall-inducing species, Gynaikothrips uzeli and Gynaikothrips ficorum. SNA Research Conference. 51: 153-155.

Williams, D \& Watson, G. 1988 The scale insects of the tropical South Pacific region. Part 1 The armoured scales (Diaspididae). CAB International. Wallingford, UK. 
INSECTOS FITÓFAGOS ASOCIADOS A Ficus benjamina L. Y A Ficus microcarpa L. EN LIMA, PERÚ Agosto - Diciembre 2013

Wille J. 1943. Entomología Agrícola del Perú. Ed. Junta de Sanidad Vegetal, Dirección Gral. de Agricultura,

Ministerio de Agricultura, Lima, Perú.

${ }^{1}$ Departamento de Entomología. Facultad de Agronomía. Universidad Nacional Agraria La Molina. Apartado 456, Lima 100, Perú. E-mail: mnarrea@lamolina.edu.pe.

${ }^{2}$ Departamento de Entomología. Facultad de Agronomía. Universidad Nacional Agraria La Molina. E-mail: cvc@lamolina.edu.pe.

3 Museo de Entomología Klaus Raven Büller - Universidad Nacional Agraria La Molina. E-mail: jmalpartida@lamolina.edu.pe. 